\title{
SAVOIRS NON FORMELS OU APPRENTISSAGES IMPLICITES ?
}

\author{
Daniel Jacobi ${ }^{1}$
}

\section{1. École et école parallèle}

La culture savante est-elle produite par l'éducation scolaire seule ? Un individu peut-il apprendre hors l'école ? Et si l'on parvient à enrichir sa culture après la période d'enseignement obligatoire, comment le fait-on ? Peut-on s'instruire seul ou par d'autres moyens que ceux des leçons ou exercices en classe ? Autant de questions presque inconvenantes tant le rôle de l'école est devenu massif. Les sociétés contemporaines ont vu, en effet, se manifester un développement sans précédent de l'école, c'est-à-dire des institutions académiques qui ont pour seule mission d'enseigner et de faire apprendre quelque chose à différentes catégories d'enfants, de jeunes ou d'adultes (école maternelle, école primaire, collège, lycée, grande école, université...). C'est cette grande mission que l'on désigne habituellement du nom d'éducation formelle pour la distinguer de toute autre forme d'intervention éducative.

\footnotetext{
1 Professeur à l'Université d'Avignon, Directeur du "Laboratoire culture \& communication".
} 


\subsection{La prépondérance de l'école}

On a observé, au cours du dernier demi-siècle, deux tendances caractéristiques de l'éducation formelle : une augmentation considérable de la population scolarisée et un allongement conséquent de la durée des études. Et ce n'est pas prendre grand risque que d'affirmer que l'école, et au-delà toutes les institutions scolaires qui constituent l'appareil d'éducation, sont devenues, dans notre société, non seulement le premier outil de socialisation (comme le notait Durkheim, il y déjà longtemps), mais aussi l'instance première de diffusion des savoirs et de la culture ${ }^{1}$.

La place et le poids de l'école sont tels qu'il est devenu presque impossible d'analyser une quelconque déficience ou lacune culturelle sans d'abord en rechercher les causes dans son fonctionnement. Les adultes n'aiment guère l'art et sont peu musiciens ? C'est parce que l'on a supprimé ces enseignements à l'école. La culture scientifique du citoyen moyen est incertaine, il communique mal en langues étrangères ? C'est que l'enseignement n'est ni adapté, ni modernisé... et ainsi de suite.

\subsection{D'autres sources d'acculturation?}

Le poids attribué à l'école et à l'appareil d'éducation formelle dans la genèse des conduites et des intérêts des adultes insérés socialement et professionnellement a complètement fait perdre de vue une donnée pourtant très connue et, par ailleurs, parfois dénoncée par les professionnels de l'enseignement : il existe d'autres sources de diffusion et d'acculturation dans une société moderne. Ces sources sont puissantes et souvent concurrentes, au point que Friedman, et plus tard Porcher, les avait appelées l'école parallèle. On songe au livre, au disque, à la presse, à la radio, au cinéma, à la télévision, aux musées et au dernier né : le multimédia. Au rôle et à l'impact de ces médias, comme à leurs contributions aux processus d'acculturation, les anglo-saxons ont donné le nom de : informal education. Expression que nous avons préféré traduire en français par éducation non formelle ${ }^{2}$.

1 E. DuRKHeIM, L'évolution pédagogique en France, Paris, Alcan, 1938.

2 D. JACOBI et B. SCHIELE, "Vulgarisation scientifique et éducation non formelle", Revue française de pédagogie, $\mathrm{n}^{\circ} 91,1990, \mathrm{pp} .81-111$. 


\subsection{Le domaine non formel}

Les caractéristiques du large secteur, aux contours indécis, que constitue l'éducation non formelle sont hétérogènes et ne peuvent être résumées de façon synthétique. Les acteurs de l'éducation non formelle, par exemple, ne sont pas astreints, comme les enseignants, à respecter les obligations et les contraintes scolaires. Il est impossible de les assimiler à des enseignants ${ }^{1}$. Les médias sont entrés, et parfois depuis longtemps, dans le secteur marchand de l'économie et dépendent de financements hétérogènes comme les ressources de la publicité. Ils se soucient en permanence de leur audience puisqu'ils ne disposent que rarement de publics captifs, etc.. Bref, sur tous les plans, l'éducation non formelle se différencie nettement de 1'enseignement académique ${ }^{2}$.

\section{Savoirs formels et non formels}

L'éducation non formelle désigne donc toutes les interventions à caractère éducatif qui sont mises en ouvre par des institutions ou des acteurs autres que l'appareil scolaire officiel. Autrement dit, la notion d'éducation non formelle ne peut être définie précisément, ou mieux construite, que par contraste avec le déroulement des apprentissages visant à favoriser l'appropriation des savoirs formels, c'est-à-dire des savoirs scolaires. Toute hypothèse concernant les savoirs non formels repose donc sur cette opposition et suppose, en préalable, une clarification de la nature des savoirs scolaires.

\subsection{Les savoirs scolaires}

Les savoirs formels sont par définition les savoirs dispensés par l'école. Depuis longtemps, cependant, les chercheurs en sciences sociales ont insisté sur l'idée que des savoirs sont construits et transmis ailleurs que pour ou dans l'école. On a donné à ces savoirs des appellations comme savoirs endogènes, savoirs chauds ou savoirs sociaux précisément pour marquer qu'ils n'appartenaient pas à la

\footnotetext{
1 D. JACOBI, "Les dispositifs non scolaires d'acculturation : introduction à l'analyse de l'éducation non formelle", Pour, n 165 , "Éducations, société", 2000.

2 D. JACOBI, La communication scientifique, Grenoble, PUG, 1999.
} 
sphère de la rationalité scientifique et technique relayée par l'école ${ }^{1}$. Mais revenons à l'école.

Toutes les institutions scolaires partagent, en effet, un certain nombre de principes et de modalités de fonctionnement qui les réunissent en un ensemble homogène. Elles sont spécialisées dans leur fonction d'éducation et sont libérées de toute autre préoccupation ; elles emploient des professionnels diplômés et qualifiés qui n'ont que cette activité à accomplir; elles sont soumises à un ensemble de règles et d'obligations communes qui sont, de plus, imposées nationalement (conditions d'accès, programmes, horaires, évaluation, délivrance de diplômes...).

\subsection{Sur l'origine du scolaire}

D'où proviennent donc les savoirs formels dispensés par l'école? La définition commune de la notion de savoir précise pourtant que la connaissance peut être acquise aussi bien par l'étude que par l'expérience.

Un détour par le processus d'élaboration des savoirs formels ou scolaires est nécessaire. Or, on sait que cette question fait débat parmi les spécialistes de l'éducation ${ }^{2}$. Sans vouloir entrer dans leurs discussions, on peut considérer que les savoirs considérés comme formels (et qu'il serait possible de désigner aussi du nom de savoirs académiques) ont comme fondement d'avoir été façonnés à des fins didactiques. Ils tirent leur force de leur capacité de s'adapter parfaitement à leur but, voire même du fait qu'ils facilitent le fonctionnement des dispositifs scolaires.

Par exemple, comment l'enseignement de l'orthographe et l'exercice de la dictée, activités académiques banales, se sont-elles généralisées ? Et pourquoi continuent-elles à occuper des élèves tout au long de leur présence à l'école ? Pourtant, l'orthographe n'a pas de fondement savant et aucune théorie scientifique de la langue écrite n'a précédé la diffusion des normes d'une graphie standard imposée et universelle. Certes, les exigences techniques de l'imprimerie, puis celles de la diffusion du livre ne sont pas pour rien dans cette

1 P. Bourdieu, Le sens pratique, Paris, Éd. de Minuit, 1980. G. Delbos, P. Jorion, La transmission des savoirs, Paris, MSH, 1990. C. GRIGNON, L'ordre des choses; les fonctions sociales de l'enseignement technique, Paris, Éd. de Minuit, 1971.

2 A. Chervel, "L'histoire des disciplines scolaires", Histoire de l'éducation, $\mathrm{n}^{\circ} 38$, mai 1988 , pp. 59-119. 
formalisation de règles et de normes, de préférence aux usages. Mais l'orthographe tire sa force de ses exigences propres en tant qu'activité d'apprentissage canonique, facile à mettre en œuvre ; et la dictée occupe le temps de la classe en même temps qu'elle discipline les apprenants ${ }^{1}$.

\subsection{Propriétés des savoirs formels}

Au-delà de cet exemple, on a pu démontrer que l'apprentissage dans des situations d'enseignement caractéristiques de l'acquisition des savoirs scolaires est généralement défini par cinq paramètres distincts bien identifiés.

2.3.1. Un savoir formel correspond toujours à un objectif et/ou une capacité (une habileté) parfaitement identifiée et résultant du travail de la transposition didactique. C'est-à-dire, pas seulement du seul travail personnel d'un maître, mais d'une élaboration lente et complexe dans laquelle interviennent différents facteurs et acteurs du champ éducatif (ce que Chevallard a proposé de nommer la noosphère) $)^{2}$.

2.3.2. Un savoir correspond à un découpage conceptuel ou une construction cohérente proposant une représentation provisoire et conventionnelle à un niveau adapté de formulation. Cette représentation destinée à être comprise, apprise et répétée est à la fois stable et dogmatique. Elle est définie et spécifiée dans un programme connu de tous (élèves et parents), documents eux-mêmes spécifiés par des instructions adressées aux maîtres. Tous, ensuite, élèves, enseignants et parents, se réfèrent à ces programmes bâtis en des curriculums intangibles.

2.3.3. L'acquisition et l'appropriation d'un savoir nécessitent l'adhésion de l'apprenant et sa participation, souvent collective et dans un cadre spécifique (la classe), à une activité précise, à la fois consciente et volontaire, généralement soutenue et régulée par un

1 J.-C. FORQUIN, "Savoirs scolaires, contraintes didactiques et enjeux sociaux", Sociologie et sociétés, Savoirs institués, savoirs informels, vol. $23, \mathrm{n}^{\circ} 1$, Presses de l'U. de Montréal, Montréal, 1991, pp. 25-40.

2 Y. ChevallaRd, La transposition didactique; du savoir savant au savoir enseigné, Grenoble, La pensée sauvage, 1985. 
maître ou un tuteur. Ce qui suppose que l'appropriation d'un savoir formel soit le résultat d'activités ou d'exercices adaptés de sorte que la répétition et l'entraînement soient organisés jusqu'à ce que l'habileté soit parfaitement acquise.

2.3.4. Comme ces savoirs sont par nature conventionnels et que les apprendre est une obligation, le maître a aussi pour fonction de prendre en charge un travail particulier sur l'éveil du désir d'apprendre et la recherche de motivations. Le public aux prises avec les savoirs formels est un public dit captif pour qui la fréquentation des institutions scolaires est une obligation ou une nécessité.

2.3.5. Enfin tous les apprentissages de ces savoirs sont formellement évalués pour juger, si possible de façon objective, qu'ils sont acquis ou maîtrisés. L'administration de ce jugement, pendant ou à l'issue de l'apprentissage, pèse fortement sur le déroulement des séquences et la nature des relations instaurées au sein du groupe classe.

\subsection{Pour définir les savoirs non formels}

En caractérisant les savoirs académiques scolaires, on peut, par comparaison point par point, tenter de dresser les spécificités des savoirs non formels, c'est-à-dire qui ne sont pas pris en charge par l'appareil scolaire et qui n'ont pas été didactisés ou transposés à des fins d'enseignement académique.

2.4.1. Les buts des savoirs non formels ne sont pas fixés a priori. Ils dépendent parfois de l'actualité ou d'intentions plus vagues comme la diffusion ou la vulgarisation pour lesquelles des savoir-faire professionnels tiennent lieu de pétition pédagogique. Ces savoirs sont dispensés par des institutions qui ont le plus souvent des buts diversifiés et pas seulement éducatifs. Par exemple, les médias visent tout autant à informer et à distraire qu'à éduquer. Les musées et les expositions scientifiques prétendent non seulement diffuser les sciences mais aussi faire jouer ou détendre.

2.4.2. Les contenus proposés ne font l'objet que d'un travail préalable minimal, par exemple de reformulation en fonction de l'image des publics visés. De plus, il n'existe évidemment pas de 
programme obligé : un média, par exemple, aborde les thèmes qu'il juge lui-même intéressants pour ses publics ou il se contente de suivre au jour le jour l'actualité.

2.4.3. Le contexte d'exposition propre à l'éducation non formelle est fort différent du cadre scolaire. Il est changeant (en famille, avec des amis, au domicile ou ailleurs...) et il est individuel ou en petit groupe. Relevant du temps libre ou du loisir, ses formes d'organisation ou sa durée sont très hétérogènes, le plus souvent imprévisibles et en général pas ou peu soutenues par les adultes. Ce qui induit par conséquent qu'il est difficile de savoir de quoi résulte l'appropriation d'un savoir non formel. Seul le travail préalable de mise en forme, des choix rédactionnels ou éditoriaux opérés avant la diffusion et qui ne peuvent être modifiés facilement en fonction des réactions du public peuvent être décrits ou analysés. Mais sont-ils réellement les générateurs d'acquisitions non formelles?

2.4.4. Le public qui choisit librement de lire, regarder, consulter ou visiter est dit spontané pour bien marquer le fait que cette décision ne relève pas d'une obligation par opposition au public contraint, ou captif, de l'école. Il ne faut cependant pas idéaliser cette opposition : un contrat implicite de communication est établi entre les producteurs et les exigences et goûts du public ; la préoccupation de fidélisation tend à stabiliser les formes des messages; des genres apparaissent et tendent à se rigidifier. Enfin, l'usage des médias et la maîtrise des moyens et supports de diffusion supposent une utilisation répétée générant des activités élaborées, mais apprises seul et sans méthode standardisée et automatisées jusqu'à ce que le sujet parvienne à en retirer ce qu'il en attend. Sans compter que les médias, eux aussi, génèrent des formes de sociabilité, mal connues et rarement décrites, mais ayant probablement des effets collatéraux.

2.4.5. Enfin, il est bien connu que l'évaluation pédagogique est exclue du champ de l'éducation non formelle. Pourtant, les effets en retour de la réception sur la production, même s'ils sont d'une tout autre nature, ne sont pas absents. Les rapports de dépendance des médias avec le système marchand, la concurrence partout présente et la nécessité capitalistique de la recherche du profit, induisent des formes de séduction ou de construction d'audience. Même si les apprentissages ne font pas l'objet d'une évaluation formelle, on sait 
qu'ils génèrent, néanmoins, une culture ou un renforcement ou plus simplement le plaisir de satisfaire une passion.

\section{Des savoirs aux apprentissages}

Ce parallèle entre des formes de savoirs ne doit pas faire perdre de vue une autre caractéristique de l'enseignement formel : à l'école, l'emphase est tout autant mise sur le contenu savant que sur les modalités particulières d'apprentissage propres à chaque discipline. Ici, il faut mémoriser par cœur ; là, disserter de façon rhétorique ; ou encore, tracer une représentation graphique, résoudre un problème selon un algorithme standard... Enseigner consiste à entraîner systématiquement des apprenants à maîtriser ces habiletés.

\subsection{Comment les savoirs non formels sont-ils acquis ?}

Toujours par contraste, comment peut-on identifier les formes d'apprentissages de l'éducation non formelle ? Deux hypothèses ont été a priori avancées : soit les sujets mobiliseraient de façon libre et volontaire les habiletés intellectuelles qu'ils ont acquises dans le cadre scolaire, soit ils utiliseraient des formes plus ou moins sophistiquées d'auto-apprentissage, capacités acquises en pratiquant les activités qui les intéressent le plus et auxquelles ils consacrent beaucoup de leur temps ou de leur énergie ${ }^{1}$. Mais, à vrai dire, aucune recherche importante ne s'est donnée les moyens de vérifier si ces hypothèses de bon sens avaient une certaine validité. C'est pourquoi, il est probablement utile d'en tester une troisième, relativement nouvelle, et qui repose sur le modèle de ce que certains psychologues appellent la mémoire implicite $^{2}$.

Comme on ne sait pas quels sont les types d'apprentissages qui sont sollicités dans l'éducation non formelle, il est tentant de penser que cette forme de mémoire autoriserait des apprentissages implicites.

1 A.M. LUCAS, "Interactions between formal and informal sources of learning science", in EVERED \& O'CONNOR (ed.), Communicating Science to the Public, Londres, J. Wiley, 1987, pp. 64.79.

2 A.S. REBER, Implicit learning and tacit knowledege; an essay on the cognitive unconscious, New York, Oxford University Press, 1993. 


\subsection{Des différences de savoir ou d'apprentissage ?}

En tentant de définir et d'identifier ce qui différencie les savoirs non formels des savoirs scolaires, on débouche sur deux voies distinctes. D'un côté, on identifie d'autres types de savoirs; de l'autre, des façons différentes d'apprendre. Ces apprentissages, en outre, se feraient sans effort, un peu comme s'ils étaient effectués de façon inconsciente, ou en tout cas à propos d'autres activités dont les buts ne sont pas pédagogiques ou didactiques.

Les modalités elles-mêmes d'organisation des apprentissages des savoirs formels dans le cadre scolaire stipulent une systématisation explicite de l'apprentissage. L'apprentissage d'un savoir formel fait l'objet d'une organisation spécifique (la situation d'apprentissage) centrée sur un objectif précis. Ce qui conduit à proposer à l'apprenant une tâche consciente et bien délimitée. Ces tâches qui correspondent à des activités d'enseignement habituellement collectives correspondent à des apprentissages tout à fait explicites : ces derniers sont mentionnés et même parfois décrits et détaillés dans les instructions et les programmes; ils sont précisés et transmis aux enseignants et on insiste auprès des élèves pour qu'ils les distinguent et s'entraînent pour chacun d'eux ${ }^{1}$.

\subsection{Le paradigme de mémoire implicite à l'épreuve}

La notion d'implicite a déjà, par le passé, été mobilisée par ceux qui s'intéressent à l'éducation non formelle. Mais les recherches récentes lui confèrent, dorénavant, un sens bien plus précis. Du point de vue des neurosciences, trois catégories de preuves sont avancées pour défendre l'existence d'une mémoire implicite ${ }^{2}$.

3.3.1. La première série de preuves prend appui sur le fait, connu depuis longtemps, qu'un certain nombre d'acquisitions psychomotrices ou purement cognitives sont en fait acquises sans qu'aucun apprentissage explicite soit prévu, organisé ou pris en charge par un éducateur ${ }^{3}$. Parmi celles-ci, la plus souvent citée, parce qu'il est

1 L.L. JACOBY, "A process dissociation framework; separating automatic from intentional uses of memory", Journal of Memory \& Language, $\mathrm{n}^{\circ} 30,1991$, pp. 513-541.

2 S. Nicolas, P. PERRUChET, "La mémoire implicite ; une vue d'ensemble", Psychologie française, vol. 43, $\mathrm{n}^{\circ} 1,1998$, pp. 3-12.

3 F.A. YATES, L'art de la mémoire, Paris, Gallimard, 1975. 
extraordinairement important pour le développement de l'intelligence, est l'apprentissage par le jeune enfant de sa langue maternelle. Sans effort et sans leçon, le tout jeune enfant acquiert la capacité à communiquer à l'aide d'un lexique qui découpe le monde et de phrases grammaticalement bien formées alors même qu'il ne les a jamais entendues ${ }^{1}$.

3.3.2. La seconde série a été tirée de recherches en neurophysiologie conduites sur des patients amnésiques ou qui, par suite d'une maladie, ou d'une lésion cérébrale, soit ont perdu l'usage de la mémoire épisodique, soit ne parviennent plus à récupérer une trace mnésique. On a démontré que ces patients continuaient néanmoins à apprendre ce qui laisse donc supposer l'existence d'une autre modalité de mémorisation qui relaierait ou remplacerait celle qui fait défaut chez eux.

3.3.3. Troisième série d'arguments en faveur de la mémoire implicite, des paradigmes expérimentaux ont été imaginés par les cogniticiens spécialiste de la mémoire. Parmi ceux-ci, le plus connu est la fabrication d'épreuves basées sur des grammaires dites artificielles. Il s'agit d'épreuves de raisonnement construites de façon totalement aléatoire de sorte qu'elle empêche le sujet de repérer une quelconque régularité ou d'abstraire et inférer une relation de causalité dans les séries qu'on lui demande d'interpréter. Or, dès la deuxième répétition de la même épreuve, on observe que les scores de celui qui répond progressent nettement alors même qu'il est persuadé de ne toujours rien comprendre à ce qu'on lui demande de faire.

\subsection{Mémoire implicite et apprentissage non formel}

La mémoire implicite fonctionnerait donc de façon très précoce chez le tout jeune enfant et se maintiendrait ensuite. Elle a été ignorée et demeure mal connue parce que cachée, toujours inconsciente, elle fonctionnerait à l'insu du sujet. Elle est localisée différemment dans le cerveau puisqu'elle persiste alors même que la mémoire explicite volontaire ne peut plus être mobilisée. Elle résiste davantage à

1 N.C. Ellis (ed.), Implicit and explicit learning of languages, Londres, Academic Press, 1994. 
l'amnésie et au vieillissement ce qui suggère qu'elle aurait un caractère permanent.

$\mathrm{Si}$, par inférence, on considère que chacune des deux formes de mémoire produit des formes distinctes de savoirs, on peut supposer que les savoirs implicites ont un caractère plus stable que les savoirs explicites. Au contraire des savoirs scolaires explicites qui, en dépit de leur masse, sont oubliés très facilement, les savoirs implicites pourraient bénéficier d'un ancrage plus fort et quasi permanent.

\subsection{Des apprentissages implicites ?}

L'hypothèse de l'existence d'autres modalités d'apprentissage (dit apprentissage implicite) prend donc appui sur la présence d'une mémoire implicite ${ }^{1}$. Celle-ci se caractériserait par la création d'une empreinte mnésique à l'insu du sujet et par la récupération inconsciente de l'information -ainsi stockée involontairement- en mémoire. En quelque sorte, nous apprenons sans arrêt et bien plus que nous ne pouvons le dire. À partir de la notion de mémoire implicite, certains auteurs ont proposé de postuler l'existence d'un apprentissage implicite. Ce dernier se distinguerait de l'apprentissage explicite propre aux situations scolaires.

\section{Les apprentissages implicites dans l'exposition}

Si l'on adhère aux notions de mémoire et apprentissage implicites, on est rapidement conduit à envisager de façon un peu différente l'opposition contrastée entre savoirs formels et savoirs non formels.

\subsection{Les caractéristiques de l'apprentissage implicite}

Pour résumer les principales qualités de l'apprentissage implicite, rappelons qu'il a tout d'abord un caractère incident. Ce mécanisme fonctionne sans effort apparent et semble en quelque sorte "naturel". Comme l'apprentissage implicite se déroule à l'insu du sujet, il apparaît indépendant, et de la conscience, et de la volonté du sujet. Par conséquent, la connaissance acquise demeure implicite dans

1 T. Meulemans, "Apprentissage implicite, mémoire implicite et développement, Psychologie française, vol. 43, $\mathrm{n}^{\circ} 1,1998$, pp. 27-37. T. MEulemans, L'apprentissage implicite; une approche cognitive, neuropsychologique et développementale, Marseille, Solal, 1998. 
le sens où elle est inaccessible à la conscience du sujet (il ne sait pas qu'il a appris quelque chose).

Les spécialistes de la mémoire implicite insistent, ensuite, sur l'idée que la structure apprise a un caractère complexe et ne correspond donc pas uniquement à des stimulations générées par des perceptions élémentaires périphériques ou secondaires. Certains d'entre eux suggèrent que l'apprentissage implicite génère des souvenirs de nature différente de ceux acquis explicitement. En contrepartie, un savoir implicite est davantage dépendant du contexte dans lequel il s'est produit. Il est, par conséquent, beaucoup moins transférable que ne le sont les apprentissages explicites.

Enfin, cette forme d'apprentissage est plus stable et plus permanente que l'apprentissage explicite. Ce qui suggère que l'apprentissage implicite est aussi plus rigide et moins adaptable à d'autres situations.

\subsection{Apprendre indirectement dans l'exposition}

La description des différentes caractéristiques de l'apprentissage implicite conduit ainsi à reconsidérer les modalités de la reconnaissance d'une exposition par les visiteurs. Certaines des caractéristiques de la mémoire implicite suggèrent sans ambiguité que l'activité de visite située dans le cadre de l'éducation non formelle se prête remarquablement à cette forme d'apprentissage.

Pour le visiteur qui parcourt librement une exposition, seul ou accompagné, le discours de l'exposition offre l'occasion d'un apprentissage au caractère incident. Celui-ci peut se produire alors que le sujet semble absorbé par d'autres préoccupations. Les intentions manifestes et les plus apparentes de l'exposition peuvent certes provoquer des effets. Mais tout autant que d'autres caractéristiques plus secondaires et que les concepteurs eux-mêmes de l'exposition n'auront ni prévues, ni imaginées. Les études d'évaluation conduites auprès des visiteurs des expositions révèlent très fréquemment ces effets implicites et non prévus.

\subsection{Le cas de la lecture}

La question du registre scriptovisuel de l'exposition et de l'activité de lecture se prête remarquablement au questionnement sur la part de la mémoire implicite. Qu'il s'agisse de la consultation de la 
signalétique, de la lecture des étiquettes ou de celle des panneaux, les enquêtes faites classiquement à la sortie semblent indiquer qu'une minorité de visiteurs fait l'effort de les lire. Pourtant, toutes les observations fines (et particulièrement celles qui sont conduites à proximité des unités d'exposition qui attirent le plus les visiteurs) prouvent, au contraire, que des modalités singulières de lecture sont bien mises en œuvre par la majorité des visiteurs.

Pour interpréter ces résultats, il est d'abord indispensable de mieux décrire et analyser les documents scriptovisuels exposés au regard des visiteurs ${ }^{1}$. Il est utile également de revenir sur les idées naïves relatives à la supériorité des images ${ }^{2}$. Et enfin, il est nécessaire de clarifier la diversité des activités lexiques que recouvre le mot lire ${ }^{3}$. Ces précautions ayant été prises, on peut montrer que la lecture est une activité de base associée à la visite de l'exposition ${ }^{4}$. Le caractère automatique et irrépressible de la lecture explique pourquoi, alors même que le visiteur interrogé affirme ne pas avoir lu les textes, certaines des indications écrites affichées influencent, malgré tout, les réponses des visiteurs. Par exemple, un titre gravé dans le fronton d'entrée d'une salle (trace témoignant d'un usage ancien et différent) peut parfaitement influencer la reconnaissance de l'exposition temporaire que cette salle historique accueille.

\subsection{Reconnaître des objets ou interpréter le discours de l'exposition?}

Dans certaines recherches, nous avons essayé de modifier une partie des textes affichés pour chercher à mesurer l'influence des textes sur la reconnaissance du discours de l'exposition ${ }^{5}$. Dans ce cas,

1 D. GaOnaC'H, J.-M. Passerault, "Marquage de l'importance et traitement des éléments dans un texte ; effets immédiats sur la lecture", European Journal of Psychology \& Education, vol. 5, n 1, 1990, pp. 59-68.

2 F. Bresson, "Compétence iconique et compétence linguistique", Communications, $\mathrm{n}^{\circ}$ 33, Seuil, 1981, pp. 185-196.

3 M.B. AlT, S.A. GRIGGS, "Psychology and the museum visitor", in J. THOMPSON (ed.), Manual of curatorship ; a guide to museum practice, Londres, Butterworth's, 1984, pp. 386-393.

4 P. MCMANUS, "Oh yes they do ; how museum visitors read labels and interact whith exhibit texts", Curator, vol. 32, $\mathrm{n}^{\circ} 3$, 1989, pp. 174-189. P. McManus, "Watch your language! People do read labels", ILVS Review, vol. 1, n² 2, 1990, pp. 125-137.

5 D. JACOBI, M.-S. Poli, Les Champs de la ville; variantes de textes, versions d'expo, Rapport de recherche non publié, Grenoble, Musée Dauphinois, 1993. 
on fabrique en quelque sorte deux versions de la même exposition identiques en tous points, sauf quant à leur registre textuel, puisqu'il est l'un des dispositifs le plus facile à modifier. Ces modifications sont évidemment faites dans une direction précise et pensée à l'avance, de sorte que l'on puisse inférer en quoi les qualités du texte influencent (ou n'influencent pas) la nature des activités de reconnaissance des visiteurs.

Dans le cas de la recherche conduite au musée Dauphinois, nous avions réécrit les étiquettes de trois dioramas qui jouaient un rôle clef pour comprendre le propos général de l'exposition, c'est-à-dire comment les rapports entre une grande ville et son espace agricole périurbain ont évolué entre 1900 et aujourd'hui. Les textes modifiés se sont, sans surprise, révélés bien plus efficaces que les textes initiaux, aussi bien auprès des publics captifs (des étudiants de l'université), que des visiteurs spontanés.

Cependant, dans le questionnaire administré à l'issue de la visite, on proposait aussi aux visiteurs interrogés des photos d'objets ethnographiques présents dans les trois dioramas. Or, on observe que les visiteurs qui n'ont pas compris quel est le propos général de l'expo, et ce aussi bien avec la version initiale qu'avec la version remaniée, se révèlent bien plus habiles que les autres visiteurs pour reconnaître les objets. Un peu comme si, en l'absence de tout autre possibilité de reconnaissance du discours de l'exposition, l'activité implicite était, pour eux, l'identification des objets comme on le fait dans toute exposition classique.

\section{Savoir non formel et apprentissage implicite}

Compte tenu de ce qui vient d'être rappelé à propos de cette forme de mémoire, il serait bien trop schématique d'attribuer au seul secteur non formel le monopole de l'implicite. Si la mémoire implicite est bien telle que les théoriciens la dépeignent, elle fonctionne en permanence et donc aussi, bien entendu, à l'école. Ce sont donc d'autres considérations qui peuvent conduire à lui attribuer une place plus ou moins importante dans l'un ou l'autre secteur. Nous suggérerons, sans prétendre être exhaustif, trois cas types qui font qu'elle est davantage sollicitée dans un cadre non formel. 


\subsection{Un territoire propice}

Il est évident que ce type d'apprentissage trouve un territoire plus favorable dans les domaines qui ne sont pas (ou qui sont mal) pris en charge par l'apprentissage explicite. Ainsi, les capacités socioaffectives, l'acquisition du goût ou de la culture esthétique se prêtent davantage à ce type d'apprentissage, ne serait-ce que parce que les institutions d'éducation éprouvent des difficultés à les assumer. Au contraire, ce sont précisément plutôt des visées de ce type que poursuivent les équipements culturels ou muséographiques. En présentant des tableaux, de beaux objets ou des spécimens rares, ils cherchent tout autant à provoquer des émotions et de la délectation qu'à transmettre des connaissances.

\subsection{Une activité favorable à l'accommodation}

Si la mémoire épisodique et la mémoire procédurale peuvent également faire l'objet d'apprentissages implicites, il est clair que les connaissances procédurales, par leur caractère opératoire, représentent un enjeu plus décisif que la seule mémorisation de savoirs déclaratifs. Or, la visite d'une exposition ou la consultation d'un dispositif interactif, sont des activités complexes, de nature psychomotrice, dans lesquelles l'orientation spatiale, les activités perceptivo-motrices et les habiletés intellectuelles sont mobilisées synergiquement. L'absence de préconisation ou de guidage par des tuteurs contraignent les visiteurs à rechercher intuitivement les potentialités des dispositifs comme leurs usages.

\subsection{L'automatisation d'une conduite experte}

Enfin, il n'est pas inutile de remarquer la convergence de la notion d'implicite avec ce que les cognitivistes appellent l'automatisation d'une tâche intellectuelle complexe comme la production d'écrit ${ }^{1}$. Cette propriété permet de ne plus encombrer la mémoire de travail par des tâches répétitives désormais intégrées. Rappelons, en effet, que l'automatisation de certaines habiletés élémentaires combinées dans des tâches complexes permet à un visiteur de mettre en œuvre

1 M. FAYOL, "Comprendre ce qu'on lit ; de l'automatisme au contrôle", in J.E. GOMBert et al. (édit.), Psychologie cognitive de la lecture, Paris, PUF, 1992, pp. 73-111. 
simultanément plusieurs d'entre elles alors que, préalablement, chacune d'elles devrait normalement l'absorber complètement et l'empêcher de faire autre chose.

L'automatisation d'une activité cognitive, comme c'est le cas pour la lecture ou la production d'écrit, permet au sujet d'apprendre rapidement, d'exécuter des activités sans que celles-ci soient disponibles dans la conscience et sans qu'elles ne diminuent la disponibilité de la capacité d'attention. Ce processus qui se déclenche automatiquement ne peut pas être interrompu quelle que soit la volonté du sujet.

On peut supposer que le visiteur plus familier des expositions sait ainsi repérer des régularités, traiter des séries complexes d'informations et au besoin prévoir, alors qu'un primo visiteur est encore obligé de traiter successivement chacune d'elles et peut être découragé par l'ampleur de la tâche. La conduite dite de visite experte ne peut être comprise que par une combinaison particulière d'automatisation et d'implicite.

En dépit de l'aspect elliptique des exemples évoqués dans ce texte, on devine qu'il est tout à fait fécond d'appliquer le modèle de la mémoire implicite à des exemples tirés du monde de l'exposition et des musées. Comme pour beaucoup d'autres domaines de recherche, la mémoire (ou l'apprentissage) implicites conduisent dorénavant à reconsidérer des données d'observation ou même des résultats de recherche. 